\title{
Síndrome hemolítico urémico en Chile: presentación clínica, evolución y factores pronósticos
}

\author{
Pedro Zambrano $0^{1,2}$, Angela Delucchi $\mathrm{B}^{3}$, Felipe Cavagnaro $\mathrm{S}^{4}$, \\ Pilar Hevia J ${ }^{5}$, María Pía Rosati $\mathbf{M}^{5}$, Elizabeth Lagos $\mathrm{R}^{5}$, Vilma Nazal $\mathrm{Ch}^{6}$, \\ Claudia G onzález $C^{7}$, Patricia Barrera B ${ }^{7}$, Enrique Alvarez $L^{8}$, Viola Pinto $S^{1}$, \\ Paulina Salas del $\mathrm{C}^{1}$, Francisco Cano $\mathrm{Sch}^{3}$, Angélica Contreras $\mathrm{M}^{6}$, \\ Mónica Galanti de la $P^{6}$, Juan Cristóbal $G$ ana $A^{3}$, Julio Zamorano $C^{9}$, \\ Amelia Espinoza $B^{10}$ Patricia D reves $R^{11}$, Jaime Pereira $\mathbf{M}^{12}$, \\ Antonia Bidegain $\mathrm{S}^{13}$, Ema Pasten $\mathrm{P}^{14}$, Leticia Yáñez $\mathrm{P}^{15}$, \\ Verónica Cerda $\mathrm{F}^{16,2}$, Eugenio Rodríguez $\mathrm{S}^{3}$, Marlene Aglony $\mathrm{I}^{3}$, \\ Elisa Gutiérrez $I^{9}$, Francisca Salas $P^{10}$, Sonia Figueroa $Y^{13}$, \\ Marcela Valenzuela $A^{13}$, Jean Grandy $H^{8}$, Boris G uerra $A^{\prime 8}$, \\ Michelangelo Lapadula A ${ }^{15}$, Paula Reutter de la $\mathbf{M}^{2}, 16$, Vivian G allardo $\mathrm{T}^{17}$, \\ Douglas Maldonado $\mathbf{S}^{\mathbf{1 7}}$, Marta Azócar $\mathrm{P}^{3}$, Gabriel Cavada $\mathrm{Ch}^{\mathbf{1 8}}$. \\ Hemolytic-uremic syndrome in Chile: clinical \\ features, evolution and prognostic factors
}

Background: Hemolytic-uremic syndrome (HUS) is characterized by acute renal failure, microangiopathic hemolytic anemia and thrombocytopenia. Aim: To describe the characteristics of patients with the diagnosis of HUS in Chile, and to identify the most reliable early predictors of morbidity and mortality. Material and methods: The clinical records of patients with HUS aged less than 15 years, attended between January 1990 and December 2003 in 15 hospitals, were reviewed. Demographic, clinical, biochemical, hematological parameters, morbidity and mortality were analyzed. Results: A cohort of 587 patients aged 2 to 8 years, $48 \%$ males, was analyzed. Ninety two percent had diarrhea. At the moment of diagnosis, anuria was observed in $39 \%$ of the patients, hypertension in $45 \%$ and seizures in $17 \%$. Forty two percent required renal replacement therapy (RRT) and peritoneal dialysis was used in the majority of cases (78\%). The most frequently isolated etiological agent was Escherichia coli. Mortality rate was $2.9 \%$ in the acute phase of the disease and there was a positive correlation between mortality and anuria, seizures, white blood cell count (WCC) $>20.000 / \mathrm{mm}^{3}$ and requirements of renal replacement therapy $(p<0.05$ ). Twelve percent of patients evolved to chronic renal failure and the risk factors during the acute phase were the need for renal replacement therapy, anuria, WCC $>20.000 / \mathrm{mm}^{3}$, seizures and hypertension. Conclusions: The present study emphasizes important clinical and epidemiological aspects of HUSin a Chilean pediatric population (Rev Méd Chile 2008; 136: 1240-6).

(Key w ords: Escherichia coli; Hemolytic-uremic syndrome; Kidney failure, acute)

Recibido el 23 de noviembre, 2007. Aceptado el 20 de junio, 2008.

${ }^{1}$ Unidad de Nefrología, Hospital de Niños Dr. Exequiel González Cortés, Santiago de Chile. ${ }^{2}$ Departamento de Pediatría, Facultad de Medicina, Universidad de Los Andes, Santiago de Chile. ${ }^{3}$ Unidad de Nefrología, Hospital Dr. Luis Calvo Mackenna, Santiago de Chile. ${ }^{4}$ Unidad de Nefrología Pediátrica, Hospital Clínico Pontificia Universidad Católica, Santiago de Chile. 5 Unidad de Nefrología Infantil, Hospital San Juan de Dios, Santiago de Chile. ${ }^{6}$ Unidad de Nefrología, Hospital Roberto del Río, Santiago de Chile. ${ }^{7}$ Unidad de Nefrología Infantil, Hospital Sótero del Río, Santiago de Chile. ${ }^{8}$ Unidad de Nefrología Infantil, Hospital Clínico San Borja Amiarán, Santiago de Chile. ${ }^{9}$ Unidad de Nefrología Infantil, Hospital Gustavo Fricke Viña del Mar, V Región. ${ }^{10}$ Servicio de Pediatńa, Hospital Regional de Coyhaique, XI Región. ${ }^{11}$ Servicio de Pediatría, Hospital Regional de Temuco, IX Región. ${ }^{12}$ Servicio de Pediatría, Hospital Regional de Chillán, VIII Región. ${ }^{13}$ Servicio de Pediatría, Hospital Regional de Concepción, VIII Región. ${ }^{14}$ Servicio de Pediatría, Hospital de Carabineros, Santiago de Chile. ${ }^{15}$ Servicio de Pediatría, Clínica Santa María, Santiago de Chile. ${ }^{16}$ Servicio de Pediatría, Hospital Parroquial de San Bemardo, Región Metropolitana. ${ }^{17}$ Departamento de Pediatría, Campus Sur, Universidad de Chile, Santiago de Chile. ${ }^{18}$ Escuela de Salud Pública, Facultad de Medicina Universidad de Chile, Santiago de Chile.

Correspondencia a: Dr. Pedro Zambrano O. Román Díaz 11 Dpto.

22, Providencia, Santiago. Fonos: 56 (2) 2360050 - 56 (9) 5380144.

Fax: 56 (2) 3629011. E mail: phzambrano@hotmail.com 
$E^{1}$ síndrome hemolítico urémico (SHU) se caracteriza por daño renal agudo, anemia hemolítica microangiopática y trombocitopenia ${ }^{1,2}$. La lesión histológica predominante es la microangiopatía trombótica (MAT), la misma que también se observa en otras enfermedades ${ }^{3}$, por lo que actualmente se sugiere su clasificación en dos grupos: a) con etiología conocida y b) con asociaciones clínicas 4 .

En pediatría, la mayoría de los casos son de origen infeccioso, precedidos de un síndrome diarreico agudo el cual es causado principalmente por una variedad de Escherichia coli enteropatógena productora de verotoxina (ECEH), cuya virulencia está dada por la presencia de un fago similar al de Shigella dysenteriae (shiga toxina) lo que motivó su nombre. La cepa más frecuente aislada en el mundo es la E coli O157:H7; otros serotipos menos frecuentes son E coli O111, O26, 055, 0103 y 0128. Shigella dysenteriae tipo I se ha asociado a la enfermedad en algunas áreas endémicas ${ }^{5,6}$.

Varias publicaciones han asociado características clínicas y parámetros de laboratorio como predictores de mortalidad y evolución desfavorable desde el punto de vista renal, pero sus resultados continúan siendo controvertidos ${ }^{7-10}$.

El objetivo de este estudio fue conocer las características clínicas, evolución, factores pronósticos de daño renal al año del diagnóstico y mortalidad en pacientes pediátricos portadores de SHU en varios centros de salud de Chile.

\section{PACIENTES Y MÉTOdO}

Se realizó un análisis retrospectivo de las fichas de pacientes menores de 15 años con diagnóstico de SHU en 15 centros de Chile que representan a 6 regiones del país entre enero de 1990 y diciembre de 2003. Se analizaron: variables demográficas, estacionalidad, síntomas previos al ingreso; presentación clínica (presencia de diarrea, compromiso neurológico, síntomas respiratorios). Parámetros de laboratorio (nitrógeno ureico en sangre (BUN), creatininemia, hemograma, recuento de plaquetas) y evolución clínica (diuresis, presión arterial, requerimientos de terapia dialítica, convulsiones, transfusiones). Se consideró diagnóstico de SHU la coexistencia de anemia hemolítica microangiopática, trombocitopenia y daño renal agudo demostrado como sedimento de orina con proteinuria o hematuria, aumento de BUN mayor a $35 \mathrm{mg} / \mathrm{dL}$ o creatinina plasmática mayor a dos desviaciones estándar por edad ${ }^{11}$. Se definió como anuria a una diuresis menor a $50 \mathrm{ml} /$ $\mathrm{m}^{2}$ /día; se consideró hipertensión arterial (HTA) a una presión arterial sobre el percentil 95 para edad y sexo, según tablas de Task Force 198712; proteinuria al índice proteína/creatinina en orina de muestra aislada mayor a 0,21 ó $>4 \mathrm{mg} / \mathrm{m}^{2} / \mathrm{h}$ en orina de $24 \mathrm{~h}$. Se consideró insuficiencia renal crónica, la depuración estimada de creatinina (eClCr) menor a $90 \mathrm{ml} / \mathrm{min} / 1,73 \mathrm{~m}^{2}$, por más de 3 meses de evolución, calculado por fórmula de Schwartz ${ }^{13-15}$. El estudio etiológico se realizó a través de cultivos de deposiciones, no se realizó búsqueda de toxinas en deposiciones ni serología.

Estadística. Los resultados se expresaron como mediana, porcentajes, desviación estándar y rangos para las variables clínicas. Prueba de chi cuadrado para variables independientes y regresión logística para identificar factores de riesgo. Se consideró estadísticamente significativo un $\mathrm{p}$ $\leq 0,05$, error alfa $5 \%$, intervalo de confianza de $95 \%$, los datos se procesaron en programa Excel y se utilizó el programa Stata 7.0.

\section{RESULTADOS}

Datos demográficos y clínicos al ingreso. Desde enero de 1990 hasta diciembre de 2003 se estudiaron 587 pacientes con diagnóstico de SHU, 48\% (n: 281) fueron de sexo masculino; la edad promedio fue 1,9 años de edad (rango: 2 meses a 8 años). El 52\% (n: 305) había recibido tratamiento antibiótico antes del ingreso. Las características demográficas, clínicas y distribución estacional se observan en la Tabla 1; la distribución según año de presentación se muestra en la Figura 1.

Evolución intrahospitalaria. Del total de pacientes, 39,5\% (n: 232) presentó anuria; 42,4\% (n: 249) requirió terapia de reemplazo renal (TRR); $78 \%$ (194/249) diálisis peritoneal; 5\% (13/249) hemodiálisis; 4\% (9/249) hemodiafiltración y 13\% (33/ 249) utilizó más de una terapia de reemplazo renal. En 4 pacientes se realizó plasmaféresis. 
Tabla 1. Características demográficas y clínicas de pacientes con diagnóstico de SH U

\begin{tabular}{|ll|}
\hline Sexo & $281(48 \%)$ hombres \\
Edad & 1,9 años $(0,17-8$ años) \\
& $0-2$ años: $374(64 \%)$ \\
& $2-4$ años: $158(27 \%)$ \\
Diarrea & $>5$ años: $55(9 \%)$ \\
Síndrome disentérico & $542(92 \%)$ \\
Días de evolución previos al diagnóstico & $349(64 \%)$ \\
Estación del año & 6 días $(1$ a 15 días) \\
& Verano: $130(31 \%)$ \\
& Otoño: $145(25 \%)$ \\
& Invierno: $102(17 \%)$ \\
& Primavera: $160(27 \%)$ \\
\hline
\end{tabular}

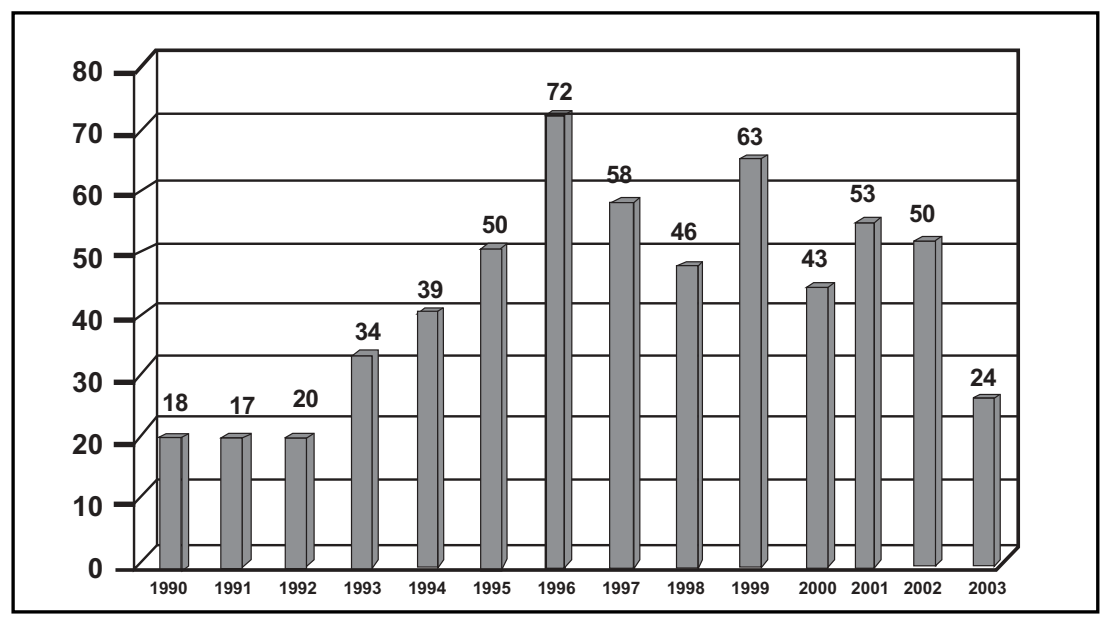

Figura 1. SHU. Número de pacientes; distribución por año.

El 22\% (n: 130) tuvo leucocitos en sangre menor a $10.000 / \mathrm{mm}^{3} ; 53 \%$ (n: 309) entre $10.000 \mathrm{y}$ 15.000 leucocitos $/ \mathrm{mm}^{3}$ y $25 \%$ (n: 148) presentó leucocitosis mayor a 20.000 .

El 44,6\% (n: 262) de los pacientes presentó HTA al ingreso; y 26,4\% (n: 155) permaneció hipertenso al alta. El agente etiológico se logró identificar en deposiciones, sangre u otros fluidos sólo en 14\% de los casos (n: 82) siendo la E coli el germen más frecuentemente aislado $(75,6 \%)$; seguido por Shigella dysenteriae (18,3\%); Streptococcus pneumoniae $(4,9 \%)$, y en un paciente se aisló Citrobacter freundii (1,2\%).

En 27\% (n: 157) de los pacientes se observaron complicaciones, la mayoría de carácter infeccioso: peritonitis (11\%); infección urinaria (9\%), bronconeumonía (3\%), septicemia (2\%), empiema y artritis séptica (2\%); 10 pacientes $(1,7 \%)$ requirieron intervención quirúrgica por cuadro compatible con abdomen agudo, evidenciándose invaginación intestinal en 6 y necrosis intestinal en 4. La mortalidad en etapa aguda de la enfermedad fue de 2,9\% (n: 17); $88 \%$ (15/17) por falla orgánica múltiple, de los otros 2 , un paciente falleció por status convulsivo refractario a tratamiento y otro por hiperkalemia refractaria al tratamiento.

Seguimiento al año. Al año del diagnóstico solamente $60 \%$ de los pacientes seguía en control (346/ 
587), 84\% (n: 290) con función renal normal sin proteinuria, un $10 \%$ (n: 36) evolucionó con eClCr menor a 90; y 6\% presentó función renal normal con proteinuria o hipertensión arterial (n: 20) (Figura 2). El 4,1\% (n: 16) se mantuvo hipertenso al año de seguimiento.

Los factores asociados a mortalidad e insuficiencia renal crónica al año de diagnóstico se observan en las Tablas 2 y 3 . Al aplicar el modelo de regresión logística, se observó que la mortalidad se asoció solamente a convulsiones (p 0,0001; RR 4,9 - 48,10), mientras que la evolución hacia la insuficiencia renal crónica (IRC), resultó ser estadísticamente significativa con la necesidad de diálisis en la etapa aguda ( $p$ 0,0001; RR 2,65 - 23,86) y la presencia de HTA ( $p$ 0,009 ; RR 1,32 - 6,95).

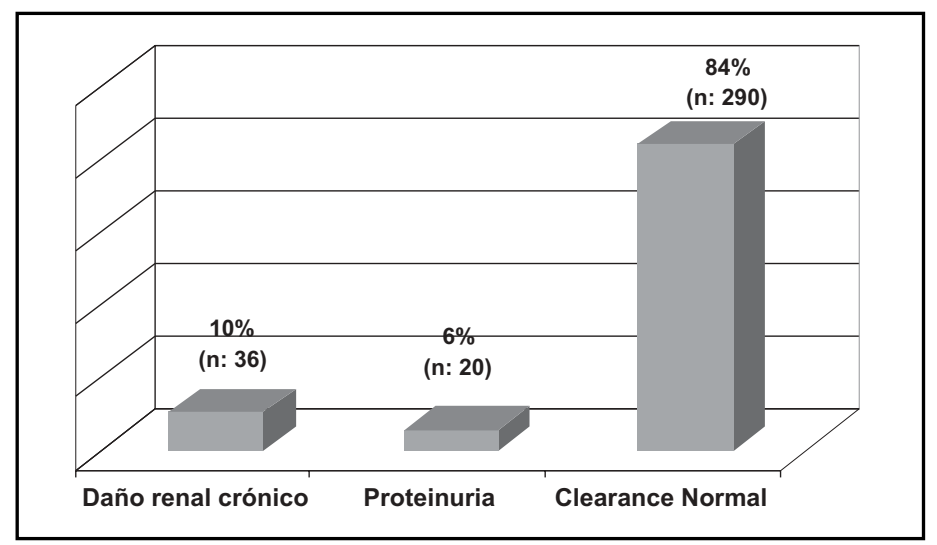

Figura 2. Evolución de la función renal al año de diagnóstico del SHU.

Tabla 2. Factores pronósticos de mortalidad en el síndrome hemolítico urémico

\begin{tabular}{|lll|}
\hline Mortalidad & $\mathrm{P}$ & $\mathrm{RR}$ \\
\hline Requerimiento de terapia de reemplazo renal & 0,0047 & $0,96-15,08$ \\
Anuria & 0,03 & $0,96-5,0$ \\
Leucocitos $>20.000$ & 0,02 & $0,93-8,52$ \\
\hline
\end{tabular}

$\mathrm{RR}$ : riesgo relativo

$\mathrm{p} \leq 0,05$

Tabla 3. Factores pronósticos entre SH U y daño renal crónico

\begin{tabular}{|lll|}
\hline IRC & $\mathrm{P}$ & $\mathrm{RR}$ \\
\hline Requerimiento de terapia de reemplazo renal & 0,0001 & $2,4-13,91$ \\
Anuria & 0,002 & $1,3-6,7$ \\
Leucocitos $>20.000$ & 0,019 & $1,0-4,9$ \\
HTA & 0,0001 & $4,0-46,56$ \\
Convulsiones & 0,002 & $1,3-6,2$ \\
\hline
\end{tabular}

IRC: Insuficiencia renal crónica. HTA: hipertensión arteria. RR: riesgo relativo $\mathrm{p}=0,05$ 


\section{DisCUSIÓN}

En Chile, el SHU es una causa frecuente de insuficiencia renal aguda e importante causa de insuficiencia renal crónica, con una incidencia de 3,2/1.000.000 de pacientes menores de 4 años (Comunicación verbal, Dra. Valeria Prado, Profesor y Sub-Director del Programa de Microbiología y Micología, Instituto de Ciencias Biomédicas, Facultad de Medicina, Universidad de Chile. XLIII Congreso Chileno de Pediatría 2003). Según datos de la rama de nefrología pediátrica de la Sociedad Chilena de Pediatría a 2004, 10,2\% de las causas de IRC corresponden a esta etiología (Comunicación verbal, Dra. Pilar Hevia, Nefróloga Infantil Hospital San Juan de Dios. Congreso Conjunto de las Sociedades de Nefrología, Hipertensión y Trasplante 2007). De los pacientes pediátricos chilenos trasplantados renales $8 \%$ tiene como causa de IRC terminal esta etiología ${ }^{16}$. A pesar de los datos antes mencionados, el SHU aún no es una enfermedad de notificación obligatoria.

Sobre SHU en Chile existen reportes aislados $^{17-20}$ que sólo muestran experiencias locales que pueden no reflejar la realidad nacional. Este reporte constituye la serie más numerosa y extendida en nuestro país e intenta reflejar la realidad nacional.

La presentación clínica de esta enfermedad no ha cambiado en los últimos 27 años ${ }^{17,18}$. El 92\% de los pacientes tuvo pródromo de diarrea, siendo en su mayoría disentérica (64\%); estas cifras son similares a lo reportado en la literatura ${ }^{7,21}$. El SHU sin pródromo de diarrea no tiene patrón estacional, es de comienzo insidioso y, en comparación con el SHU clásico, tiene mayor frecuencia de secuelas como proteinuria, hipertensión arterial, mayor incidencia de IRC y mortalidad ${ }^{8,22,23}$. En esta revisión no se encontró diferencia en el seguimiento entre aquellos que presentaron pródromo de diarrea (o etiología conocida) y aquellos de causa desconocida; esto podría deberse a que algunos de estos pacientes no presentaron diarrea pese a tener como causa a la E coli verotoxina positiva, factor reportado en otras series $^{10}$. La baja identificación del agente etiológico podría explicarse por el frecuente uso de antibióticos previo al ingreso, sumado a la falta de medios de cultivos especiales y a la ausencia de un protocolo de estudio que incluya la búsqueda de E coli enterohemorrágica, verotoxina en deposiciones o de anticuerpos específicos en sangre.

La mortalidad de reportes internacionales varía entre $0 \%$ y $25 \%{ }^{9}, 10,17,18,24$ en nuestra serie fue de $2,9 \%$, cifra similar a las publicadas por otros autores nacionales y extranjeros 7,10,17,18. El compromiso neurológico y específicamente la presencia de convulsiones se han asociado a mayor mortalidad, lo que coincide con lo encontrado en esta serie ${ }^{25}$.

El mejor conocimiento de la fisiopatología de la enfermedad, el manejo conservador, la instalación precoz de terapia de reemplazo renal y la sospecha oportuna de complicaciones ha mejorado la sobrevida en las últimas décadas. Pese a esto, algunos de estos pacientes aún fallecen en el contexto de falla orgánica múltiple. No existe un tratamiento específico para el daño endotelial y sus consecuencias. Varios estudios muestran las características clínicas de la enfermedad y han intentado identificar factores pronósticos de morbimortalidad; la variabilidad de sus resultados puede explicarse por la heterogeneidad de los pacientes incluidos ${ }^{9,10,19-22}$; en nuestro estudio, la necesidad de diálisis fue similar a lo descrito en la literatura ${ }^{7,18,20 .}$.

La anuria, HTA y la leucocitosis se han identificado como factores de mal pronóstico desde el punto de vista renal $7,9,19$; en nuestra experiencia los resultados son similares. En esta serie, al igual a lo reportado en la literatura, 10\% evolucionó a insuficiencia renal crónica y $6 \%$ presentó evidencias de daño renal manifestado por HTA 0 proteinuria?.

El seguimiento de estos pacientes es fundamental para la detección precoz de signos de daño renal como proteinuria o hipertensión, los que pueden aparecer en el transcurso de los años. Publicaciones recientes demuestran que los pacientes que presentaron SHU evolucionaron con microalbuminuria en su seguimiento en relación a pacientes normales ${ }^{26-28}$. En ellos, la utilización de medicamentos como los inhibidores de la enzima convertidora de angiotensina o asociados a los bloqueadores de los receptores AT1 de angiotensina II han disminuido la progresión del daño renal no inmunológico ${ }^{29,30}$.

Por otra parte, de esta experiencia surge la necesidad de estudios prospectivos colaborativos orientados a la búsqueda del agente etiológico, 
conductas terapéuticas oportunas y adecuadas tanto en la fase aguda como a largo plazo para disminuir la progresión del daño renal.

Dado que numerosos animales sanos portan ECEH en el contenido intestinal y que, inevitablemente, existe algún grado de contaminación fecal de las carnes y subproductos de origen animal, la mejor forma de controlar el problema es, sin duda alguna, mejorando las condiciones higiénicas en que se procesan y manipulan los alimentos en la cocina, con especial énfasis los de origen cárnico. Es importante destacar que esta bacteria muere a temperaturas iguales o superiores a los $68,5^{\circ} \mathrm{C}$, es capaz de sobrevivir en alimentos ácidos ( $\mathrm{pH}$ igual o mayor a 2,5) por varios días y no se destruye con la congelación de los alimentos ${ }^{31}$.

\section{REFERENCIAS}

1. Von Gasser C, Gauthier E, Steck A. Siebenmann RE, Deschun R. Hämolytische-urämische Syndrome. Bilaterale nierenrinden-nekrosen bei akuten erworbenen haemolytischen Anämien. Schweiz Med Wochenschr 1955; 85: 905-9.

2. Kaplan BS, Proesmans W. The Hemolytic Uremic Syndrome and its Variants. Seminars in Hematology 1987; 24: 148-60.

3. Ruggenenti P, Noris M, Remuzzi G. Thrombotic microangiopathy, hemolytic uremic syndrome, and thrombotic thrombocytopenic purpura. Kidney Int 2001; (60): 831-46.

4. Besbas N, Karpman D, Landau D, Loirat C, Proesmans W, ReMUZZi G et al. A classification of hemolytic uremic syndrome and thrombotic thrombocytopenic purpura and related disorders. Kidney Int 2006; 70: 423-31.

5. EXENI R. Síndrome Hemolítico Urémico. Arch Latin Nefr Ped 2001; 1: 35-56.

6. Karmali MA, Petric M, Lim C, Fleming PC, Arbus GS, Lior $H$. The association between idiopathic hemolytic uremic syndrome and infection by verotoxinproducing Escherichia coli. J Infect Dis 1985; 151: 775-82.

7. SizzirRi FD, Rahman RC, Bibiloni N, Ruscasso JD, Amoreo O. Childhood hemolytic-uremic syndrome in Argentina: long term follow-up and prognosis features. Pediatr Nephrol 1997; 11: 156-60.

8. Renaud C, Niaudet P, Gagnadoux F, Broyer M, Habib R. Haemolytic uraemic syndrome: prognostic factors in children over 3 years of age. Pediatr Nephrol 1995; 9: 24-9.
Esfuerzos para prevenir la enfermedad por medio de irradiación de la carne de los animales portadores de agentes asociados a la producción de SHU en los mataderos, vacunas y agentes farmacológicos son promisorios, pero las medidas de salud pública y cuidados personales continúan siendo factores de vital importancia en la prevención de la contaminación de los alimentos como también en la transmisión de persona a persona especialmente en menores de 5 años ${ }^{32}$.

Finalmente, un programa de educación a nivel nacional, similar al realizado con el cólera sería de gran utilidad y tendería a disminuir la contaminación cruzada que es aparentemente la más frecuente en niños.

9. Mencía S, Martínez De Azagra A, De Vicente A, MonleON M, CASAdo J. Síndrome hemolítico urémico. Análisis de 43 casos. An Esp Nefrol 1999; 50: 467-71.

10. Gianviti A, Tozzi A, De Petris L, Caprioll A, Ravá L, EDEFONTI A ET AL. Risk factors for poor renal prognosis in children with hemolytic uremic syndrome. Pediatr Nephrol 2003; 18: 1229-35.

11. Goldsmith D, Novelo A. Clinical and laboratory Evaluation of Renal Function. En Edelman Chester Jr. Pediatric Kidney Disease; Boston. Little Brown and Company. 1992. p. 465.

12. Update on the 1987 Task Force Report on High Blood Pressure in Children and Adolescents: A Working Group Report from the National High Blood Pressure Education Program. Pediatrics 1996; 98: 649.

13. k/DOQI Clinical Practice Guidelines for Chronic Kidney Disease: Evaluation, Classification, and Stratification. Am J Kidney Dis 2002.

14. SchwARTZ GJ, GAUTHIER B. A simple estimate of glomerular filtration rate in adolescent boys. J Pediatr 1985; 106: 522-6.

15. Schwartz GF, FELD LG, LANGFord DJ. A simple estimate of glomerular filtration rate in full-term infants during the first year of life. J Pediatr 1984; 104: 849-54.

16. Rosati P, Pinto V, Delucchi A, Salas P, Cano F, Zambrano P et al. Pediatric renal transplantation: 13 years of experience-report from the Chilean cooperative multicenter group. Transplant Proc 2005; 37: 1569-73.

17. GonZÁlez F. Síndrome Hemolítico Urémico. En: Gasc O, y cols Nefrología en la práctica pediátrica. Santiago: Editorial Andrés Bello 1981; 113-9. 
18. Cordero J, Baeza J, Fielbaum O, Saieh C, Rodríguez E, Olivos P et al. Síndrome Hemolítico Urémico. Experiencia de 154 casos. Rev Chil Pediatr 1990; 61: 235-42.

19. Zambrano P, Delucchi A, Hevia $P$, Nazal V, Rosati $P$, BARRERA P ET al. Síndrome Hemolítico Urémico en Santiago de Chile: Evolución de la función renal y factores pronósticos. Rev Chil Pediatr 2005; 76: 48-56.

20. Cavagnaro F, Gana J, Lagomarsino E, Vogel A, GederLiNE A. Síndrome hemolítico urémico. Experiencia de un centro pediátrico. Rev Méd Chile 2005; 133: 781-7.

21. Siegler L, Pavia A, Hansen F, Christofferson R, Cook J. Atypical hemolytic-uremic syndrome: A comparison with postdiarrheal disease. J Pediatr 1996; 128: 505-11.

22. Fitzpatrick M, Walters M, Trompeter R, Dilon M, BARRAT M. Atypical (non-diarrhea-associated) hemolytic-uremic syndrome in childhood. J Pediatr 1993; 122: $532-7$.

23. Constantinescu A, Bitzan M, Weiss L, Christen E, Kaplan B, Cnaan A et al. Non-Enteropathic Hemolytic Uremic Syndrome: Causes and Short-Term Course. Am J Kidney Dis 2004; 43: 976-82.

24. Al-Eisa A, Al-Hajerr. Hemolytic Uremic Syndrome in Kuwait Arab Children. Pediatr Nephrol 2001; 16: 1093-8.

25. Garg AX, Suri RS, Barrowman N, Rehman F, Matsell D, Rosas-ARELIANo MP ET AL. Long-term renal prognosis of diarrhea -associated hemolytic uremic syndrome.
A systematic review, meta-analysis, and meta-regression. JAMA 2003; 290: 1360-70.

26. Cobeñas C, Alconcher L, SpizZirri A, Rahman R. Longterm follow-up of Argentinean patients with hemolytic uremic syndrome who had not undergone dialysis. Pediatr Nephrol 2007; 22: 1343-7.

27. Garg A, Ciark W, Salvadori M, Macnab J, Suri R, HAYNES R ET AL. Microalbuminuria three years after recovery from Escherichia coli 0157 hemolytic uremic syndrome due to municipal water contamination. Kidney Int 2005; 67: 1476-82.

28. Garg A, Salvadori M, Okell J, Thiessen-Pilbrook $\mathrm{H}$, SuRi R, Fimer G et AL. Albuminuria and estimated GFR 5 years after Escherichia coli 0157 Hemolytic Uremic Syndrome. An update. Am J Kid Dis 2008; 51: 435-44.

29. Delucchi A, Cano F, Rodríguez E, González X, Cumsilue M. Enalapril and prednisone in children with nephrotic- range proteinuria. Pediatr Nephrol 2000; 14: 1088-91.

30. Van Dyck M, Proesmans W. Renoprotection by ACE inhibitors after severe hemolytic uremic syndrome. Pediatr Nephrol 2004; 19: 688-90.

31. Borie C. Algunas consideraciones sobre la "Bacteria de la carne". Tecno Vet; 1996; 2 (2). http:// www.tecnovet.uchile.cl

32. EXENI A. Recomendaciones para el manejo del Síndrome Urémico Hemolítico. Arch Latin Nefr Ped 2003; 3: 98-100. 